\title{
Evaluation of Role of Hysteroscopic Selective Chromotubation in Proximal Tubal Blockage in Infertility
}

\author{
1Juhi Bharti, ${ }^{2}$ Banashree Das, ${ }^{3} \mathrm{HP}$ Anand
}

\section{ABSTRACT}

Aim: The aim of the study was to evaluate the role of hysteroscopic selective chromotubation in proximal tubal blockage in infertility patients.

\begin{abstract}
Materials and methods: Infertility patients with documented proximal tubal block on hysterosalpingography (HSG), after standard investigations, were enrolled in this study after proper informed written consent. After detailed work up and evaluation, all the patients underwent conventional laparoscopic chromotubation under general anesthesia. Patients with tubal blockage even after conventional chromotubation underwent hysteroscopic selective chromotubation and the results were compared. In this procedure, tubal patency of each tube was evaluated one after another by injecting the dye directly in the ostia through the cannula under hysteroscopic guidance.
\end{abstract}

Results: Out of the 27 tubes which were found to be blocked even after conventional laparoscopic chromotubation, selective chromotubation revealed free spillage of dye in $44.4 \%$ of these tubes. On comparison, only $25 \%$ tubes were patent after conventional laparoscopic chromotubation, whereas $50 \%$ tubes were patent after selective chromotubation. This difference was statistically significant $(p=0.003)$.

Conclusion: Hysteroscopic selective chromotubation is a better diagnostic test for tubal patency than conventional laparoscopic chromotubation.

Keywords: Cannulation, Fallopian tube patency tests, Infertility, Selective chromotubation.

How to cite this article: Bharti J, Das B, Anand HP. Evaluation of Role of Hysteroscopic Selective Chromotubation in Proximal Tubal Blockage in Infertility. J South Asian Feder Obst Gynae 2015;7(2):51-54.

Source of support: Nil

Conflict of interest: None

\footnotetext{
${ }^{1}$ Senior Resident, ${ }^{2,3}$ Professor

${ }^{1}$ Department of Obstetrics and Gynecology, All India Institute of Medical Sciences, New Delhi, India

${ }^{2,3}$ Department of Obstetrics and Gynecology, VMMC and Safdarjung Hospital, New Delhi, India
}

Corresponding Author: Juhi Bharti, Senior Resident Department of Obstetrics and Gynecology, All India Institute of Medical Sciences, New Delhi, India, Phone: 01129575101 e-mail: drjuhigeorgian.04@gmail.com
Date of received: 15 May 2015

Date of acceptance: 20 June 2015

Date of publication: August 2015

\section{INTRODUCTION}

Tubal pathology is among the most common cause of infertility and it accounts for approximately 30 to $35 \%$ of all infertility cases. The incidence of tubal factor infertility varies from place to place. In India, it accounts for roughly $40 \%$ of total infertility cases. ${ }^{2}$ Therefore, tests to determine tubal patency bear an important place in evaluation, diagnosis and treatment of infertility.

Tubal diseases can be proximal or distal. For the therapeutic approach, it is important to distinguish among irreversible obstruction (e.g. fibrotic stenosis), reversible obliteration (plugs) and simple spasm. Tubal patency test was first described by Rubin ${ }^{3,4}$ in 1920 using carbon dioxide $\left(\mathrm{CO}_{2}\right)$. Since then, numerous methods have been developed for evaluation of tubal patency. Tubal patency can be diagnosed by hysterosalpingography (HSG) or laparohysteroscopy with chromotubation, saline infusion sonography, and hysterosalpingo-contrast sonography (HyCoSy).

In hysteroscopic selective chromotubation, dye is directly pushed into the tube after cannulating the ostia. It is a known fact that if one tubal lumen is blocked due to kinking of the tube or small mucous plug or debris, it will give false negative result. Again if one tube is patent and other tube is blocked by debris or mucous plug, dye will flow through patent tube and will give negative result for that tube. So, in selective chromotubation, the increased pressure generated by the direct injection helps to overcome obstructions associated with mucous plugs or minor synechiae.

Accurate diagnosis of tubal patency is necessary to avoid unnecessary in vitro fertilization procedures or microsurgical tubal anastomosis. This study was done keeping in mind that laparohysteroscopic selective chromotubation may help in accurate diagnosis and proper management of patients with tubal factor infertility. It may also have therapeutic value in minor obstruction of tubes. 


\section{MATERIALS AND METHODS}

After taking approval from the ethics committee of the institute, the present study was conducted at a tertiary care center. Twenty-four patients with documented unilateral or bilateral proximal tubal blockage on HSG were included in the study. After detailed work up and evaluation, all the patients underwent conventional laparoscopic chromotubation under general anesthesia. The patients with evidence of tubal blockage even after conventional laparoscopic chromotubation underwent laparohysteroscopic selective chromotubation.

In conventional chromotubation, diluted methylene blue (around $15 \mathrm{cc}$ ) was injected into the cavity of the uterus using Leech Wilkinson cannula and spillage of the dye was observed laparoscopically. In the patients where there was no evidence of spillage of dye in either of the tube, operative hysteroscope (Tekno Brand, Germany) was introduced. A small caliber $(1.6-1.8 \mathrm{~mm})$ polyethylene cannula was introduced through operating channel and tubal ostia was identified. This cannula was passed into the tube for about 3 to $4 \mathrm{~mm}$ and about 10 to $15 \mathrm{cc}$ of diluted sterile methylene blue was injected through it (Fig. 1). Presence or absence of spillage of the dye/change of contour of the tube was observed laparoscopically. Same procedure was repeated in the other tube also where necessary.

Statistical analysis was done using software SPSS-17. Total number of patients in whom selective chromotubation resulted in free peritoneal spill of dye (positive result) was analyzed against total number of patients who had negative result in initial chromotubation. The tests of statistical significance used to analyze same parameter between two variables were Chi-square test and Fisher's exact test. Binomial test was used for statistical significance between different variables of same parameter.

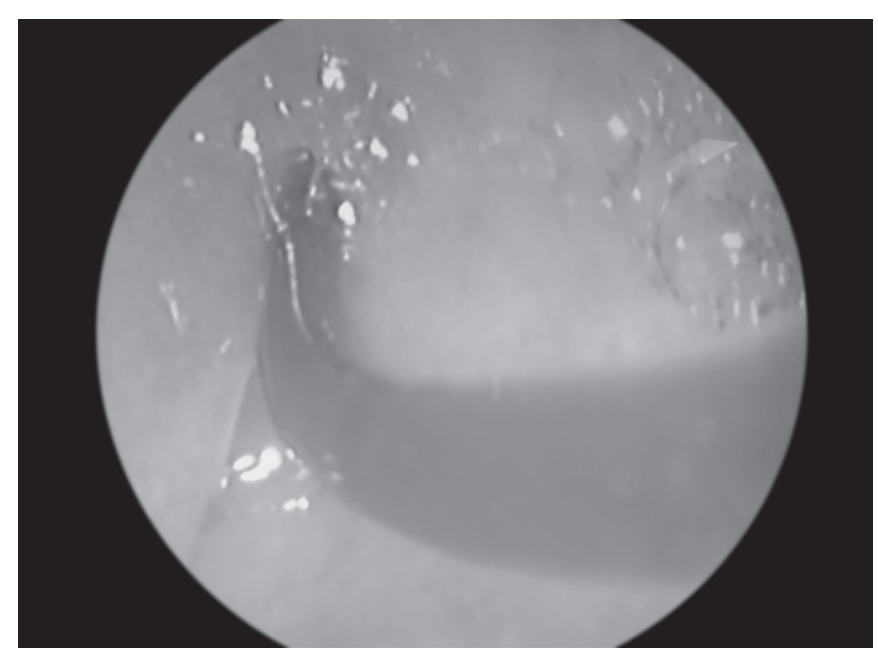

Fig. 1: Hysteroscopic selective chromotubation: Dye going freely in the ostia via flexible cannula
Table 1: Tubal patency on conventional chromotubation

\begin{tabular}{lll}
\hline & Number & Percentage \\
\hline Patent & 10 & 24.4 \\
Blocked & 31 & 75.6 \\
\hline Total & 41 & 100 \\
\hline
\end{tabular}

Table 2: Tubal patency on selective chromotubation

\begin{tabular}{lll}
\hline & Number & Percentage \\
\hline Patent & 12 & 44.4 \\
Blocked & 15 & 55.6 \\
\hline Total & 27 & 100 \\
\hline
\end{tabular}

\section{RESULTS}

The mean age of the patients was $28.96 \pm 3.12$ years. Of the 24 patients, primary infertility was present in 12 $(50 \%)$ patients, whereas rest of the $12(50 \%)$ patients had secondary infertility. The duration of infertility ranged from 2 to 15 years with a mean of $6.4 \pm 3.6$ years.

Out of the 24 patients included in the study, 20 patients had bilateral tubal blockage, whereas four had unilateral tubal blockage. So, the total number of tubes included in the study was 44 . Two patients were excluded from the study due to non visualization of uterus and tubes because of dense adhesions. Out of these, one patient had history of previous salpingectomy. So, conventional laparoscopic chromotubation was done for evaluation of 41 tubes in a total of 22 patients. Out of these 41 tubes, 10 tubes $(24.4 \%)$ were found to be patent with free spillage of dye. So, 31 tubes $(75.6 \%)$ were confirmed to have blockage even after conventional chromotubation (Table 1).

Selective chromotubation was attempted for evaluation of rest of the 31 tubes. But, the procedure could not be performed in two patients (4 tubes) as the ostia could not be visualized on hysteroscopy due to reasons like fibroid polyp and adhesions. In the remaining 27 fallopian tubes which were evaluated by selective chromotubation, free spillage of dye was seen in 12 tubes (44.4\%) (Table 2).

On comparison, $50 \%$ of tubes were patent after selective chromotubation, whereas only $25 \%$ tubes were patent after conventional chromotubation. $\mathrm{p}$-value for this comparison was 0.003 which was statistically significant.

\section{DISCUSSION}

In vitro fertilization (IVF) procedure is the only option left for patients with bilateral tubal blockage. In vitro fertilization procedures, though a boon for infertility patients in the present era, is costly and not affordable by every patient, especially in a developing country. The success 
rate is also not very promising. It gives emotional and psychological stress to the patients. Therefore, accurate diagnosis of tubal patency is must for proper management of infertility patients. There has always been a quest for ideal tubal patency test. Several methods have evolved for evaluation of tubal factors. Hysterosalpingography and laparoscopic chromotubation are the most commonly employed tests. The present study was undertaken with an aim to evaluate the efficacy of selective chromotubation both as diagnostic and therapeutic procedure. This procedure can be combined with the conventional laparoscopic chromotubation.

There are only two studies found in the literature where similar procedure was used. In a study published in 2006, Wongtra-Ngan $S^{5}$ found that the overall success rate was $12 / 18$ tubes (66.7\%). In their study, out of seven patients with bilateral tubal obstruction, procedure was successful bilaterally in four patients and unilaterally in three patients. They have advocated it not only as better diagnostic procedure but mainly as alternative treatment for proximal tubal occlusion.

In a recent study done in 2010 by Szabo I, ${ }^{6}$ a total of 67 hysteroscopies with selective chromotubation was performed. His procedure was successful in $66.67 \%$ of cases even when bilateral occlusion of the fallopian tubes was diagnosed by previous HSG or laparoscopic chromotubation while in cases of unilateral occlusion this was achieved in $80.95 \%$ of cases.

The success of these studies can be best evaluated by pregnancy outcome among the patients. In our study, three patients got pregnant during 3 months follow-up. One patient had bilateral tubal blockage on HSG and conventional laparoscopic chromotubation. But, after selective chromotubation, both her tubes came out to be patent. This patient had infertility for 6 years with one previous abortion. She conceived on ovulation induction and delivered a healthy male baby. Other patients are in follow-up and receiving ovulation induction as per protocol. No case of ectopic pregnancy was observed.

Wongtra-Ngan S ${ }^{5}$ in 2006 reported four spontaneous pregnancies in 3 (27.3\%) out of 11 patients and all were intrauterine pregnancies. The pregnancy rate in our study is lower than the above mentioned studies which can be explained by very short follow-up period in our study.

It is an emerging procedure which can not only detect tubal patency in a more accurate way but might have therapeutic effect as well. Sulak et al, ${ }^{7}$ in 1987, reported the histological findings of resected proximal tubal obstructions and found that $33 \%$ of these had only amorphous material in the lumen. In selective chromopertubation, the increased pressure generated by the direct injection helps to overcome obstructions associated with mucous plugs or minor synechiae.

On analyzing the results of tubal patency after conventional laparoscopic chromotubation in the present study, 10 out of 41 tubes (24.4\%) showed free spillage of dye which implied that HSG falsely reported blockages in $24.4 \%$. Possible explanation for false blockages in HSG could be cornual spasm, which gets relieved when laparoscopy is performed under general anesthesia. Several studies have compared HSG and laparoscopy for the diagnosis of tubal patency. ${ }^{8-11}$

\section{CONCLUSION}

Thus, we can conclude that selective chromotubation diagnoses tubal patency more accurately and should be recommended in all patients undergoing laparohysteroscopy. It should be an integral part of diagnostic work up of all infertility patients with proximal tubal blockages instead of conventional laparoscopic chromotubation. Besides diagnostic role, it may also be of therapeutic value in some patients due to flushing action of dye on amorphous tissue debris, mucous plugs, etc. when injected with increased pressure. It restores patency and consequently the fertility in such cases.

It might avoid unnecessary IVF procedure which is a financial and emotional burden to these patients. The safety and ease of the procedure make it a viable option before sending the patients to IVF center. Studies with larger sample size and long-term follow-up are required to establish its role as routine procedure in infertility work up.

\section{REFERENCES}

1. Speroff L, Fritz MA. Clinical gynaecologic endocrinology and infertility. 8th ed. Philadelphia: Lippincott Williams and Wilkins; Dec 2010:1156.

2. Kore S, Hegde A, Nair S, et al. Sonography for assessment of tubal patency: our experience. J Obstet and Gynecol India 2000;50(2):63-66.

3. Rubin IC. Nonoperative determination of fallopian tubes in sterility: intrauterine insufflation with oxygen and production of subphrenic pneumoperitoneum. JAMA 1920;74(15):1017.

4. Rubin IC. Therapeutic aspects of uterotubal insufflation in sterility. Am J Obstet Gynecol 1945;50:621.

5. Wongtra-Ngan S, Vutyavanich T. Alternative treatment for infertile patients with proximal tubal obstruction. Chiang Mai Med Bull 2006;45(3):113-118.

6. Szabo I. Comparative analysis of the clinical examination methods of proximal tubal occlusion, and evaluation of related patient satisfaction [PhD Theses]. Pécsi University, School of Med Sci Hungary; 2010. 
7. Sulak PJ. Histologies of proximal tubal occlusion. Fertil. Steril 1987;48(3):437-440.

8. Vasiljević M, Ganović R, Jovanović R, Marcovic A. Diagnostic value of hysterosalpingography and laparoscopy in infertile women. Srp Arh Celok Lek 1996;124(5-6): 135-138.

9. Naila U. Hysterosalpingography. Professional Med J Dec 2005;12(4):386-391.
10. Tankova M, Nalbanski B, Bolisa BL, Borisov S. A comparative study between hysterosalpingography in evaluating female infertility. Obstet-Gynaecol 2000;39(1):20-22.

11. Lavy Y, Lev-Sagie A, Holtzer H, Revel A, Hurwitz A. Should laparoscopy be a mandatory component of the infertility evaluation in infertile women with normal hysterosalpingogram or suspected unilateral distal tubal pathology? Eur J Obstet Reprod Biol 2004;114(1):64-68. 